\title{
RELAÇÕES ÁGUA-SOLO-PLANTA-ATMOSFERA
}

\author{
DESENVOLVIMENTO INICIAL DO ABACAXIZEIRO, \\ CV. SMOOTH CAYENNE, SOB DIFERENTES CONDIÇÕES \\ DE SALINIDADE DA ÁGUA
}

\author{
Francisco José Loureiro Marinho ${ }^{1}$, Pedro Dantas Fernandes ${ }^{2}$ e Hans Raj Gheyi ${ }^{3}$
}

\begin{abstract}
RESUMO
Foram estudados, em casa-de-vegetação, os efeitos de diferentes níveis de salinidade na água de irrigação sobre o desenvolvimento do abacaxizeiro, cv. 'Smooth Cayenne', durante os primeiros 140 dias, em um Regossolo. Adotou-se o delineamento experimental em blocos casualizados, com 4 repetições, sendo testados 8 níveis de salinidade de água (condutividade elétrica de $0,1,2,3,4,5,6$ e $7 \mathrm{dS} . \mathrm{m}^{-1}$ a $25^{\circ} \mathrm{C}$ ), preparados com a adição de $\mathrm{NaCl}$ e $\mathrm{CaCl}_{2}$, mantendo a proporção de 7:3 entre $\mathrm{Na}$ e Ca, respectivamente. Com a elevação dos níveis de salinidade da água de irrigação ocorreram reduções significativas nas variáveis estudadas (índice de enraizamento das plantas, emissão de folhas, área foliar, fitomassa das partes aérea e raiz da planta) sendo os efeitos mais drásticos observados nos tratamentos com níveis de salinidade acima de $3 \mathrm{dS} . \mathrm{m}^{-1}$.
\end{abstract}

Palavras-chave: crescimento, tolerância a salinidade, qualidade de água, Ananas comosus, L.

\section{INITIAL GROWTH OF PINEAPPLE CV. SMOOTH CAYENNE UNDER DIFFERENT WATER SALINITY CONDITIONS}

\begin{abstract}
This research evaluated the effects of different salinity levels of irrigation water on the growth of pineapple cv. Smooth Cayenne, during initial stages up to 140 days under greenhouse conditions. The soil utilized in the study was classified as Rhegossol. A randomized block experimental design, consisting of 8 levels of water salinity (electrical conductivity of $0,1,2,3,4,5,6$ and $7 \mathrm{dS} \cdot \mathrm{m}^{-1}$ at $25^{\circ} \mathrm{C}$ ) with 4 replications was adopted. The salinity levels were obtained by adding adequate volumes of $\mathrm{NaCl}$ and $\mathrm{CaCl}_{2}$ solutions, maintaining the 7:3 proportion between $\mathrm{Na}$ and $\mathrm{Ca}$, respectively. The results obtained show that high levels of salinity in the irrigation water provoked significant reductions in the studied variables (establishment index, leaf emission, leaf area, weights of areal and root parts) with the most drastic effects observed in treatments with salinity levels of irrigation water above $3 \mathrm{dS} . \mathrm{m}^{-1}$.
\end{abstract}

Key words: growth, salt tolerance, water quality, Ananas comosus, L.

\section{INTRODUÇÃO}

O abacaxizeiro é uma das principais frutíferas cultivadas no Nordeste. A produção nacional no ano de 1997 foi de
1.284.211.000 frutos (Anuário Estatístico do Brasil, 1997) sendo o Estado da Paraíba responsável por, aproximadamente, $40 \%$ dessa produção.

A evapotranspiração (ETm) da cultura varia entre 700 e

\footnotetext{
${ }^{1}$ Eng. Agrônomo, Mestre, Secretaria da Agricultura Indústria e Comércio, Prefeitura Municipal de Campina Grande, Fone (083) 341-3659 Fax 083) 3226596 E-mail: Sainco@cgnet.com.br

2 Prof. Adjunto, Dr., Departamento de Engenharia Agricola, Centro de Ciências e Tecnologia, Universidade Federal da Paraíba, CP 10.087, fone (083)310-1055, E-mail: copeag@ deag.ufpb.br

${ }^{3}$ Prof. Titular, Dr., DEAg/CCT/UFPB, CP 10.087, fone (083)310-1056, e-mail: hans@ deag.ufpb.br
} 
1000mm.ano ${ }^{-1}$. Mesmo sendo a sua exigência em água, relativamente baixa, quando comparada com outras frutíferas, o abacaxizeiro é sensível ao déficit hídrico, especialmente durante o período de crescimento vegetativo, quando são determinados o tamanho e as características dos frutos (Doorenbos \& Kassam, 1994).

Segundo Reinhardt (1996) são grandes as vantagens da irrigação na abacaxicultura, garantindo menores riscos de produção, acréscimos na produtividade da cultura e melhor qualidade dos frutos.

No Nordeste brasileiro, a qualidade das águas que podem ser utilizadas em irrigação é muito variável, tanto em termos geográficos como ao longo do ano, principalmente em pequenos açudes. Com a utilização de águas com níveis mais altos de condutividade elétrica, comumente encontrados no final do período de estiagem, há sérios riscos de salinização dos solos a serem irrigados, com prejuízos, também, para as culturas (Costa \& Gheyi, 1984; Laraque 1989; Audry \& Suassuna 1990).

Segundo Kamphorst \& Bolt (1976) quando se aplica água em uma cultura, mesmo de boa qualidade, são incorporadas quantidades apreciáveis de sais ao solo; portanto, deve ser uma preocupação constante a prevenção da salinização em áreas irrigadas.

Com relação às espécies vegetais, os efeitos da salinidade e/ou da sodicidade são diferentes, variando entre espécies e entre genótipos de uma mesma espécie, visto que algumas produzem rendimentos aceitáveis, em níveis relativamente altos de condutividade elétrica, enquanto outras são mais sensíveis. Esta diferença deve-se à melhor capacidade de adaptação osmótica de algumas espécies, sendo capazes de absorver e acumular íons e utilizá-los na síntese de compostos orgânicos, além de terem maior capacidade de absorção de água, mesmo em potenciais osmóticos muito baixos. É muito útil essa variabilidade genética entre as plantas, por permitir a seleção de espécies e cultivares mais tolerantes e capazes de produzir rendimentos economicamente aceitáveis, em condições de salinidade (Ayers \& Westcot, 1991).

Várias tecnologias têm sido utilizadas para se produzir, economicamente, em condições de solo ou de água, com altos riscos de salinização; dentre elas, o uso de culturas consideradas tolerantes à salinidade tem-se destacado, sendo importantes os estudos que visem avaliar a sensibilidade das espécies ao estresse salino.

Enquanto para outras culturas são encontradas várias referências relatando os efeitos de qualidade de água e de salinidade do solo sobre os rendimentos, raros são os trabalhos sobre a cultura do abacaxi. Maas (1984) inclui esta espécie entre as medianamente tolerantes, sem apresentar qualquer índice relacionando produção e níveis de salinidade.

Sideris (1955) estudando o desenvolvimento do abacaxizeiro cultivado em regiões litorâneas, observou que as gotículas de água marítima prejudicam o desenvolvimento das plantas, com o aparecimento de necrose nas bordas das folhas.

Fonteno \& McWillians (1974) trabalhando com outras bromeliáceas, verificaram que as espécies de folhas finas são extremamente sensíveis, enquanto as de folhas largas são relativamente resistentes à salinidade.

Considerando-se a escassez de informações sobre os efeitos do estresse salino em abacaxizeiro, o presente trabalho foi realizado com o objetivo de se estudar o desenvolvimento inicial dessa espécie em condições de salinidade da água de irrigação.

\section{MATERIAL E MÉTODOS}

O trabalho foi desenvolvido em casa de vegetação, localizada no Campus II da Universidade Federal da Paraíba, Campina Grande, PB, no período de junho a outubro de 1996, utilizando-se material de um Regossolo, proveniente da região de Salgadinho, município de Campina Grande, PB. As características físicas e químicas do solo, determinadas no Laboratório de Irrigação e Salinidade do Departamento de Engenharia Agrícola/CCT/UFPB, conforme metodologias recomendadas pela EMBRAPA (1979) estão apresentadas na Tabela 1, observando-se que o mesmo possui textura arenosa, baixos valores de condutividade elétrica no extrato de saturação e de percentagem de sódio trocável.

Os tratamentos constaram de 8 níveis de salinidade da água de irrigação: $\mathrm{T}_{0}$ - testemunha, $\mathrm{T}_{1}-1 \mathrm{dS} \cdot \mathrm{m}^{-1}, \mathrm{~T}_{2}-2 \mathrm{dS} \cdot \mathrm{m}^{-1}$, $\mathrm{T}_{3}-3 \mathrm{dS} \cdot \mathrm{m}^{-1}, \mathrm{~T}_{4}-4 \mathrm{dS} \cdot \mathrm{m}^{-1}, \mathrm{~T}_{5}-5 \mathrm{dS} \cdot \mathrm{m}^{-1}, \mathrm{~T}_{6}-6 \mathrm{dS} \cdot \mathrm{m}^{-1} \mathrm{e}$ $\mathrm{T}_{7}-7 \mathrm{dS} \cdot \mathrm{m}^{-1}$. As águas foram obtidas a partir da adição, em água destilada, de cloreto de sódio e cloreto de cálcio, na proporção de 7:3, em termos equivalentes, conforme composição média de águas utilizadas na irrigação de pequenas propriedades do Nordeste (Medeiros, 1992).

O delineamento experimental foi em blocos casualizados, com 4 repetições e 3 plantas por parcela.

Foram utilizadas mudas de abacaxi (Ananas comosus, L.) cv. Smooth Cayenne, tipo rebentão, com peso médio de $170 \mathrm{~g}$ e com a maior homogeneidade possível em cada bloco. Foram plantadas em vasos plásticos contendo $9,8 \mathrm{~kg}$ de material de solo, previamente irrigado com a água do respectivo tratamento.

Tabela 1. Características físicas, hídricas e químicas do solo utilizado no experimento

\begin{tabular}{|c|c|c|}
\hline Atributos & Unidade & Valores \\
\hline Densidade aparente & g.cm ${ }^{-1}$ & 1,67 \\
\hline Classificação textural & Franco-arenoso & \\
\hline pH (pasta de saturação) & & 4,26 \\
\hline \multicolumn{3}{|l|}{ Extrato de saturação : } \\
\hline Condutividade Elétrica & $\mathrm{ds} \cdot \mathrm{m}^{-1}$ & 0,316 \\
\hline RAS & $\left(\text { mmoles.L } L^{-1}\right)^{0,5}$ & 1,14 \\
\hline Complexo sortivo: & $\mathrm{cmol}_{\mathrm{c}} \cdot \mathrm{kg}^{-1}$ & \\
\hline $\mathrm{H}+\mathrm{Al}$ & & 2,02 \\
\hline $\mathrm{Ca}$ & & 1,21 \\
\hline $\mathrm{Mg}$ & & 0,39 \\
\hline $\mathrm{Na}$ & & 0,08 \\
\hline $\mathrm{K}$ & & 0,03 \\
\hline СТC & & 3,70 \\
\hline PST & & 4,76 \\
\hline Retenção de Umidade: & $\%$ & \\
\hline $10 \mathrm{kPa}$ & & 9,73 \\
\hline $150 \mathrm{kPa}$ & & 2,14 \\
\hline
\end{tabular}


A adubação pré-plantio constou de $200 \mathrm{~g}$ de esterco de gado bovino e $10 \mathrm{~g}$ de super fosfato simples, misturados ao solo, em cada vaso. As adubações de cobertura foram realizadas a intervalos semanais, um dia após as irrigações utilizando-se, a cada vez, $0,25 \mathrm{~g}$ de $\mathrm{Ne} 0,25 \mathrm{~g}$ de $\mathrm{K}_{2} \mathrm{O}$ por planta, em soluções diluídas de $5 \mathrm{ml}$, com aplicação na axila das folhas basais.

As irrigações foram semanais, de acordo com a água evapotranspirada e o volume a ser aplicado, determinado através de pesagens em uma das repetições. Houve controle do volume das soluções utilizadas nas irrigações por tratamento. Mensalmente, o volume de água foi ajustado de modo a proporcionar uma lixiviação equivalente a $15 \%$ da água utilizada pela planta durante o período.

Além do cuidado de se escolher mudas em bom estado fitossanitário, as mesmas foram tratadas com os produtos parathion (Folisuper) - 1g.L $\mathrm{L}^{-1}$ e benomyl (Benlate) - 1g.L. $\mathrm{L}^{-1}$, para prevenção contra a cochonilha do abacaxizeiro (Dismicocus brevipes) e contra a fusariose (Fusarium moniliforme var. subglutines). Aos 45 dias do plantio, aplicouse Carbaril (pó seco) na concentração de 7,5\%, visando ao controle da broca-do-talo (Castnia icarus ).

No final do estudo, aos 140 dias pós-plantio, foi feita avaliação do índice de enraizamento das mudas (\%), do número de folhas emitidas durante o período e da área foliar. A área foliar foi estimada utilizando-se a equação $Y=2,44+12,47 x-0,24 x^{2} \quad\left(R^{2}=0,89\right)$, onde $\mathrm{x}$ representa o peso da folha, obtida por Marinho (1996) trabalhando com plantas da mesma cultivar. $\mathrm{Na}$ colheita, as plantas foram coletadas com o devido cuidado, separando-se folhas, caule e raízes para obtenção do peso da matéria seca (fitomassa) através de secagem em estufa a $60{ }^{\circ} \mathrm{C}$.

As análises estatísticas foram realizadas conforme Pimentel Gomes (1987) e determinadas equações de regressão para as variáveis em estudo, em função dos níveis de salinidade.

Houve transformação do número (n) de folhas emitidas em $(x+n)^{1 / 2}$ para fins de análise estatística, conforme recomendação de Snedecor \& Cochran (1974).

\section{RESULTADOS E DISCUSSÃO}

O aumento da salinidade da água provocou redução nos volumes utilizados nas irrigações durante o estudo (Tabela 2) devido, provavelmente, à redução nos processos de absorção e transpiração das plantas. Segundo Richards (1954) existe uma estreita relação entre a salinidade e a inibição da absorção de água pelas plantas, em virtude dos efeitos osmóticos. Santos (1990) também observou este fato na fase de desenvolvimento

Tabela 2. Volumes de água consumida, por tratamento, durante o período de estudos

\begin{tabular}{ccccccc}
\hline $\begin{array}{c}\text { Tratamentos } \\
\text { Dias }\end{array}$ & $0-30$ & $30-60$ & $60-90$ & $90-120$ & $120-140$ & Total \\
\hline $\mathrm{T}_{0}$ & 10,9 & 11,41 & 9,68 & 15,10 & 11,90 & 58,99 \\
$\mathrm{~T}_{1}$ & 9,30 & 10,35 & 8,35 & 14,45 & 11,86 & 54,31 \\
$\mathrm{~T}_{2}$ & 9,55 & 10,64 & 8,77 & 14,74 & 11,14 & 54,84 \\
$\mathrm{~T}_{3}$ & 9,23 & 10,27 & 8,14 & 14,11 & 11,51 & 53,26 \\
$\mathrm{~T}_{4}$ & 8,55 & 9,13 & 7,64 & 14,20 & 10,99 & 50,51 \\
$\mathrm{~T}_{5}$ & 8,43 & 9,25 & 7,73 & 13,98 & 11,36 & 50,75 \\
$\mathrm{~T}_{6}$ & 7,10 & 8,70 & 7,49 & 13,89 & 11,22 & 48,40 \\
$\mathrm{~T}_{7}$ & 8,62 & 8,80 & 8,03 & 14,22 & 11,38 & 51,05 \\
\hline
\end{tabular}

*Três plantas de uma repetição inicial de bananeiras, em condições de casa de vegetação, utilizando águas de diferentes tipos de salinidade. Observa-se que as diminuições foram proporcionais conforme o nível de salinidade da água, com ligeiro aumento de consumo de $\mathrm{T}_{7}$. Os volumes de água consumidos foram maiores a medida em que a planta crescia, com decréscimo entretanto, no período de 60 a 90 dias (julho a agosto) coincidindo com dias de temperatura mais baixa e menor insolação.

Pelos dados da Figura 1, verifica-se que até o nível de CEa de $3 \mathrm{dS} \cdot \mathrm{m}^{-1}\left(\mathrm{~T}_{3}\right)$, o enraizamento das mudas não foi afetado, todos os tratamentos apresentando percentuais de $100 \%$, enquanto nos tratamentos $\mathrm{T}_{4}, \mathrm{~T}_{5}, \mathrm{~T}_{6}$ e $\mathrm{T}_{7}$ os índices baixaram para 50,75, 66 e 41\%, respectivamente. Os níveis de salinidade a partir de $4 \mathrm{dS} . \mathrm{m}^{-1}$ na água de irrigação provocaram inibição no enraizamento das mudas de abacaxizeiro. As plantas no tratamento $\mathrm{T}_{4}$ apresentaram baixa percentagem de enraizamento em relação a $T_{5}$ e $T_{6}$ devido à incidência de doenças (Marinho 1996). Vale frisar que neste tratamento o consumo de água também foi menor.

Estes resultados estão de acordo com afirmações de Doneen (1975) de que a salinidade pode provocar morte das plantas na fase inicial, e com dados obtidos por Araújo Filho (1991) com diferentes cultivares de bananeira, quando verificou desuniformidade no enraizamento de mudas, em condições de salinidade.

$\mathrm{O}$ aumento da concentração salina na água de irrigação provocou redução na área foliar (Figura 2). Foi obtida a equação de regressão, significativa a nível de 0,01 de probabilidade, $Y=1380,8-96,7 x+3,4 x^{2},\left(R^{2}=0,91\right)$ relacionando-se a área

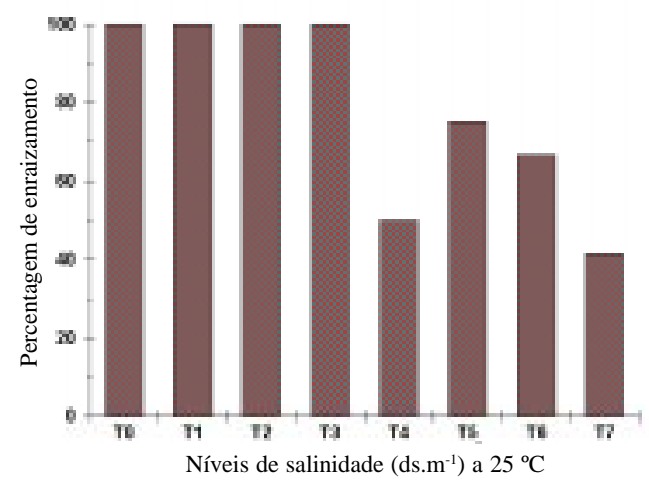

Figura 1. Índice de enraizamento das plantas em função do nível de salinidade da água de irrigação.

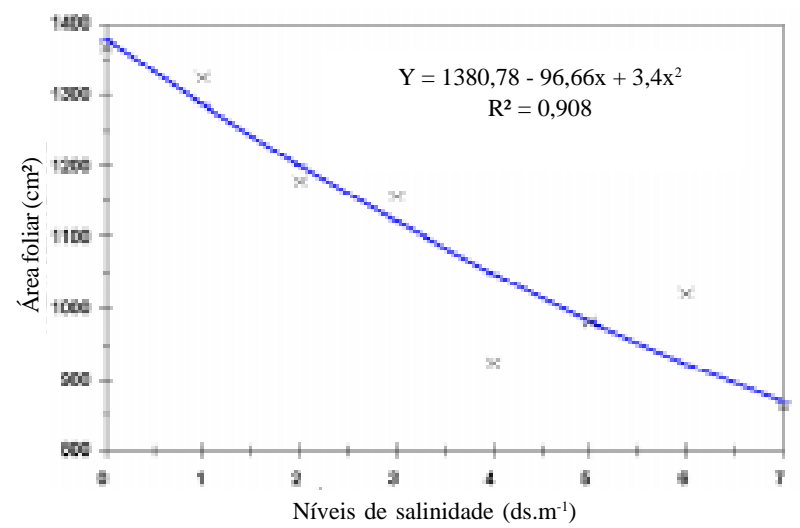

Figura 2. Área foliar das plantas de abacaxizeiro, aos 140 dias após-plantio, em função da salinidade da água de irrigação. 
foliar com níveis de salinidade de água utilizada na irrigação. Em termos relativos, comparando-se $\mathrm{T}_{0}$ com os tratamentos de $\mathrm{CEa}$ até 3,0 $\left(\mathrm{T}_{4}\right)$, as diminuições de área foliar ficaram abaixo de $15 \%$, enquanto para os tratamentos com CEa superior a 3,0 dS.m ${ }^{-1}$ as reduções foram, em média, superiores a $25 \%$, chegando a até $37 \%$ no tratamento $\mathrm{T}_{7}$ Ocorreu, também, redução no peso da matéria seca das plantas (fitomassa) como conseqüência do aumento de salinidade (Figura 3). A produção de matéria seca nos tratamentos $\mathrm{T}_{1}, \mathrm{~T}_{2}, \mathrm{~T}_{3}, \mathrm{~T}_{4}, \mathrm{~T}_{5}, \mathrm{~T}_{6}$ e $\mathrm{T}_{7}$ em relação à testemunha $\left(\mathrm{T}_{\mathrm{o}}\right)$, foi da ordem de 99,4, 92,3, 88,3, 83,8 84,3, 85 e $80,7 \%$, respectivamente. Através do estudo de regressão é possível se estimar, com razoável precisão $\left(\mathrm{R}^{2}=0,93\right)$ a fitomassa através da equação $\mathrm{Y}=26,823-1,398 \mathrm{x}+0,093 \mathrm{x}^{2}$, onde $(\mathrm{x})$ representa a condutividade elétrica da água de irrigação em dS.m $m^{-1}$ a $25^{\circ} \mathrm{C}$.

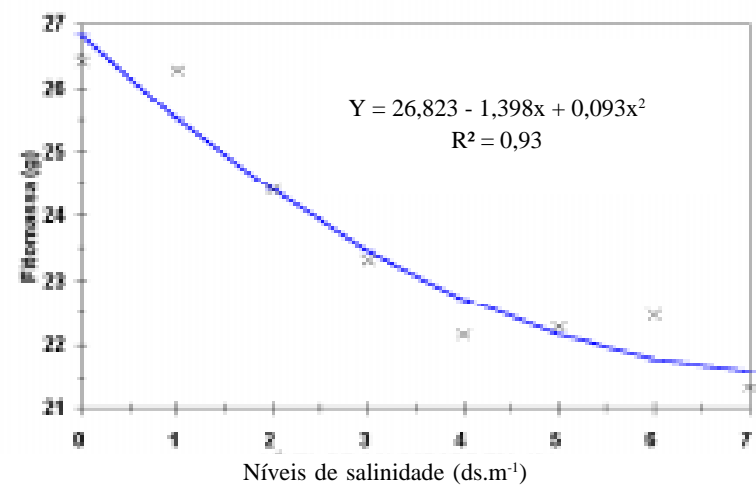

Figura 3. Fitomassa das plantas de abacaxizeiro aos 140 dias após plantio em função da salinidade da água de irrigação

O aumento da salinidade na água de irrigação, mesmo em níveis relativamente baixos, como $1 \mathrm{dS} \cdot \mathrm{m}^{-1}\left(\mathrm{~T}_{1}\right)$, provocou acentuada redução no peso da matéria seca das raízes, da ordem de $56,6 \%$. A partir do tratamento $\mathrm{T}_{4}$, a maioria das plantas teve desenvolvimento radicular muito pequeno ou inexistente. Pela equação de regressão $Y=536,8-155,5 x+12,62 x^{2}$, significativa no nível de 0,01 de probabilidade, observa-se que os valores de peso da matéria seca das raízes (Y) diminuíram com o aumento de salinidade da água de irrigação (x), o que vem sendo observado, também, nas outras variáveis. Os reduzidos valores de matéria seca das raízes, inclusive da testemunha podem, em parte, ser justificados pelas informações contidas em Domingo et al. (1992) que citam ser pequeno, superficial e compacto o sistema radicular do abacaxizeiro, com grande parte das raízes concentrada na camada superficial até $20 \mathrm{~cm}$ de profundidade.

Os valores médios de emissão de folhas pelas plantas da testemunha $\left(\mathrm{T}_{0}\right)$ após 140 dias do plantio, foram entre $65,5 \mathrm{a}$ $116 \%$ superiores em relação aos tratamentos $\mathrm{T}_{1}$ e $\mathrm{T}_{7}$. Pelos estudos de regressão verifica-se que $99 \%$ do número de folhas emitidas (Y) podem ser explicados pela seguinte equação, significativa a 0,05 de probabilidade:

$$
Y=4,15-3,06 x+2,08 x^{2}-0,62 x^{3}+0,08 x^{4}-0,004 x^{5}
$$

onde $\mathrm{x}$ representa condutividade elétrica da água de irrigação em dS.m ${ }^{-1}$ a $25^{\circ} \mathrm{C}$.

Pelas informações contidas na Figura 4 verifica-se que o número de folhas emitidas nos tratamentos $\mathrm{T}_{1}$ a $\mathrm{T}_{7}$ variou em torno de 2,5; isto indica que o abacaxizeiro, mesmo quando irrigado com água de alta salinidade, mantém-se metabolicamente ativo, embora em ritmo reduzido denotando, assim, certa capacidade de adaptação às condições salinas.

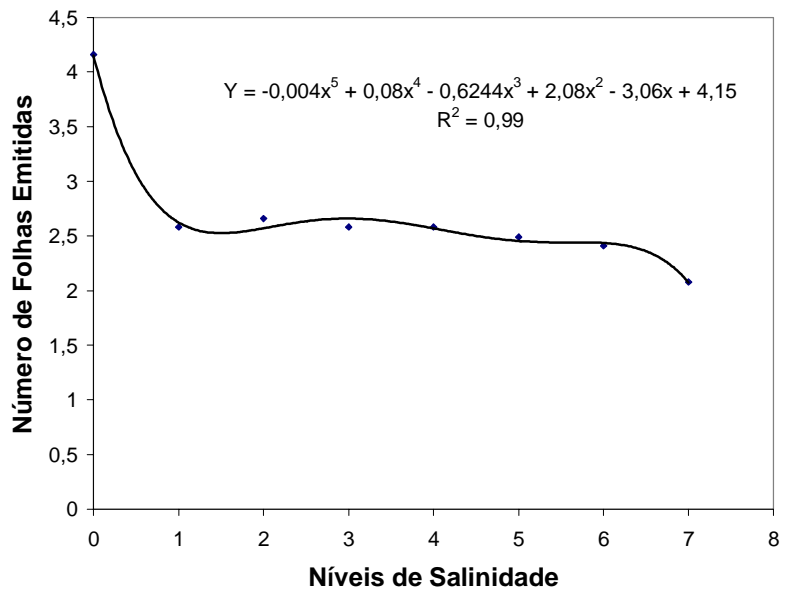

Figura 4. Número de folhas emitidas aos 140 dias após plantio, em função dos níveis de salinidade da água de irrigação

Durante a condução deste trabalho observou-se ocorrência crescente de necrose na extremidade do limbo das folhas das plantas, com aumento do teor de sais na água de irrigação, principalmente a partir de $4 \mathrm{dS} . \mathrm{m}^{-1}\left(\mathrm{~T}_{4}\right)$. Pelos dados apresentados, nota-se ter havido redução acentuada das variáveis enraizamento, emissão de folhas, área foliar e peso da matéria seca, tanto da parte aérea como das raízes, a medida em que foi aumentado o nível de salinidade da água de irrigação; entretanto, os efeitos mais drásticos foram observados no peso da raiz e no número de folhas emitidas.

Doneen (1975) relata que altas concentrações salinas afetam o metabolismo da planta, provocando mudanças na anatomia e na morfologia, com reflexos sobre o crescimento e sobre a emissão de ramos e folhas dos vegetais. Segundo Ayers \& Westcot (1991) a tolerância à salinidade pode variar de acordo com o estágio de desenvolvimento das plantas sendo, entretanto, a fase inicial a mais sensível.

\section{CONCLUSÕES}

Os resultados obtidos em condições de casa de vegetação permitem enumerar as seguintes conclusões :

1. O crescimento e desenvolvimento do abacaxizeiro cv. Smooth Cayenne são afetados com o aumento da concentração salina na água de irrigação, com efeitos mais drásticos a partir de 4 dS.m ${ }^{-1}$.

2. O enraizamento das mudas é inibido quando as plantas são irrigadas com águas que apresentam concentração salina superior a $3 \mathrm{dS} \cdot \mathrm{m}^{-1}$.

3. O aumento da concentração salina na água de irrigação provoca redução na área foliar das plantas; em níveis de 1 a 3 $\mathrm{dS} . \mathrm{m}^{-1}$ as reduções são inferiores a $15 \%$ e, em níveis superiores a $4 \mathrm{dS} \cdot \mathrm{m}^{-1}$ as reduções ultrapassam $25 \%$.

4. A fitomassa também é afetada pela salinidade da água de irrigação, com reduções superiores a $15 \%$ quando os níveis estão acima de $3 \mathrm{dS} \cdot \mathrm{m}^{-1}$. 


\section{REFERÊNCIAS BIBLIOGRÁFICAS}

ANUÁRIO ESTATÍSTICO DO BRASIL. Rio de Janeiro: IBGE, 1997.

ARAÚJO FILHO, J.B. Efeitos de diferentes níveis de salinidade de solo na composição química da folha $\mathrm{e}$ crescimento de cultivares de bananeira (Musa sp.) Campina Grande: UFPB, 1991, 87p. (Dissertação de Mestrado).

AUDRY, P.; SUASSUNA, P. A qualidade da água na irrigação do trópico semi-árido. Um estudo de caso. In: SEMINÁRIO FRANCO-BRASILEIRO DE PEQUENA IRRIGAÇÃO, 1990, Recife. Anais. ; Recife PE: 1990, p.147-153.

AYERS, R.S.; WESTCOT D.W. A qualidade da água na agricultura. Campina Grande, UFPB, 1991. 218p.

COSTA, R.G.; GHEYI, H.R. Variação da qualidade da água de irrigação da microrregião homogênea de Catolé do Rocha, PB. Pesquisa Agropecuária Brasileira, Brasília, v.19, p.1021-1025, 1984.

DOMINGO, H.R.C.; REINHARDT, D.H.R.C.; MEDINA, V.M. Crescimento e qualidade do fruto do abacaxi cvs. Pérola e Smooth Cayenne. Pesquisa Agropecuária Brasileira. Brasília, v.27 n.3 p.435-447, mar. 1992.

DONEEN, L.D. Water quality for irrigated agriculture. In: POLJAKOFF - MAYBER, A.; GALE, J. (ed). Plants in saline environments. Berlin: Springer-Verlag. 1975. p. 56-64.

DOORENBOS, J.; KASSAM, A.H. Efeito da água no rendimento das culturas. Campina Grande: UFPB, 1994. p.181-184

FAO/UNESCO. Irrigation drainage and salinity; An international sourcebook. Paris: Hutchinson, 1973. 510p.

EMBRAPA. Serviço Nacional de Levantamento e Conservação de Solos, Manual de métodos de análise de solo. Rio de Janeiro.. EMBRAPA-SNLCS, 1979. [n.p.]

FONTENO, W.C.; McWILLIANS, E.L. Factors influencing the landscape use bromeliads, Americam Society for Horticultural Science, v. 18, p. 257-278, 1974.
KAMPHORST, A., BOLT, G.H. Saline and sodic soils. In: BOLT, G.H.; BRUGGEnT, M.G.M. (eds.) Soil chemistry. Amsterdam: Elsevier Scientific Publishing Company, 1976, p-171-191. (Development in Soil Science, 5A).

LARAQUE, A. Estudo e previsão da qualidade química da água dos açudes do Nordeste. Recife : SUDENE, 1989. 97p. (SUDENE. Hidrologia, 26).

MARINHO, F.J.M. Avaliação do desenvolvimento e crescimento de mudas de abacaxizeiro (Ananas comosus) cv. Smooth cayenne, em condições de salinidade de água de irrigação. Campina Grande,PB: UFPB, 1996. 83p. (Dissertação de Mestrado)

MEDEIROS, J.F. de Qualidade de água de irrigação e evolução da salinidade nas propriedades assistidas pelo GAT nos Estados de RN, PB e CE. Campina Grande: UFPB, 1992. 173p (Dissertação de Mestrado)

MAAS, E.V. Crop tolerance. California Agriculture., v.38, n.10, p.20-4, 1984.

PIMENTEL GOMES, F. Curso de estatística experimental. 12 ed. São Paulo: Nobel, 1987. 467p.

REINHARDT, D.H. Potencial da fruticultura irrigada nos tabuleiros costeiros. Cruz das Almas, BA: EMBRAPACNPMF, 1996. 23p.

RICHARDS, L.A. Diagnosis and improvement of saline and alkali soils. Washington: United States Department of Agriculture, 1954. 160 p. (Agriculture Handbook, 60)

SANTOS, G.R. Crescimento da bananeira nanica (Musa $s p$.) sob diferentes qualidades de água de irrigação. Campina Grande: UFPB, 1990. 78p. (Dissertação de Mestrado).

SIDERIS, C.P. Effects of sea water sprays on pineapple plant. Phytopath, v.45, p.590-594, 1955

SNEDECOR, G.W.; COCHRAN, W.G. Statistical methods. Ames: Iowa State College, 1974. 593 p 